\title{
Landnutzung und biologische Vielfalt in Deutschland - Welchen Beitrag zur Nachhaltigkeit können Großschutzgebiete leisten?
}

\author{
Peter Meyer $^{1} \cdot$ Laura Demant $^{1} \cdot$ Jan Prinz $^{2}$
}

Eingegangen: 11. Januar 2016 / Angenommen: 22. August 2016 / Online publiziert: 15. September 2016

(C) Springer-Verlag Berlin Heidelberg 2016

Zusammenfassung Der land- und forstwirtschaftlichen Nutzung kommt eine Schlüsselrolle für die Erhaltung der biologischen Vielfalt zu. In diesem Zusammenhang untersuchen wir zum einen die Kennzeichen und den Status quo einer nachhaltigen Land- und Forstwirtschaft und zum anderen den Beitrag der deutschen Großschutzgebiete zur Verwirklichung einer nachhaltigen Landnutzung. Nachhaltig sind land- und forstwirtschaftliche Nutzungen, wenn sie bei der Bedarfsdeckung der heutigen Generation diejenige der kommenden Generationen nicht einschränken. Das wichtigste Kennzeichen von Nachhaltigkeit ist ein funktionierendes Managementsystem, in dem eine periodische Wirkungskontrolle und Adjustierung der Nutzung auf der Grundlage aussagekräftiger Indikatoren durchgeführt wird. Die derzeit eingeführten Indikatorensysteme sind im Hinblick auf die biologische Vielfalt noch unvollständig, weisen nicht immer Zielwerte auf und ermöglichen nur eingeschränkt Aussagen zum Zusammenwirken von Zuständen, Belastungen und Maßnahmen. Mit den Großschutzgebietskategorien in Deutschland werden unterschiedliche Ziele verfolgt. Während Nationalparke aufgrund des Ausschlusses land- und forstwirtschaftlicher Nutzung auf dem

Dr. Peter Meyer

Peter.Meyer@nw-fva.de

Laura Demant

Laura.Demant@nw-fva.de

Dr. Jan Prinz

jp@umweltplan.de

1 Sachgebiet Waldnaturschutz/Naturwaldforschung, Nordwestdeutsche Forstliche Versuchsanstalt, Grätzelstraße 2, 37079 Göttingen, Deutschland

2 Umweltplan GmbH Stralsund, Tribseer Damm 2, 18437 Stralsund, Deutschland größten Teil ihrer Fläche Vorranggebiete für den Naturschutz darstellen, dienen Naturparke und Biosphärenreservate der Integration von Nutzungs- und Schutzzielen. Die Großlandschaften Deutschlands werden durch die verschiedenen Großschutzgebiete nur eingeschränkt repräsentiert. Damit Naturparke und Biosphärenreservate zukünftig verstärkt Modellräume für die Integration von Landnutzung und Naturschutz darstellen können, sollten ihr Management verbessert und eine systematischere Schutzgebietsplanung durchgeführt werden. Bei Nationalparken ist bereits deutlich erkennbar, dass sie ihre Vorrangfunktion für den $\mathrm{Na}$ turschutz in naher Zukunft erfüllen werden.

Schlüsselwörter Nachhaltigkeit · Forstwirtschaft · Landwirtschaft · Großschutzgebiete · Biodiversität · Evaluierung $\cdot$ Indikatoren

\section{Land Use and Biological Diversity in Germany - To What Extent Can Large Protected Areas Contribute to Sustainability?}

Abstract Agriculture and forestry play a central role in preserving biological diversity. In this context, we examined the attributes and present state of sustainability of agriculture and forestry in respect of biological diversity in Germany. Additionally, we asked whether large protected areas can serve as model sites for the integration of land use and conservation or as priority areas for nature conservation. Sustainable land use is a way of managing natural resources to meet present needs without limiting future generations in fulfilling their needs. The main attribute of sustainability is the implementation of a management system that safeguards potential multifunctional use. The indicator systems implemented on the national level in Germany to evalu- 
ate sustainability of agriculture and forestry are shown to be incomplete in respect of biological diversity and do not always contain target values. With the different types of large protected areas in Germany different targets are pursued. While national parks exclude agriculture and forestry to a large extent and therefore serve as priority areas for conservation, nature parks and biosphere reserves aim at the integration of land use and nature protection. Large protected areas in Germany do not exhibit a representative spatial distribution. In order to render nature parks and biosphere reserves to model sites for the integration of land use and nature conservation their management needs to be improved considerably and an even more systematic conservation planning should be pursued. In contrast, national parks already reveal that they will fulfill their task as priority sites for nature conservation in the near future.

Keywords Sustainability · Forestry · Agriculture $\cdot$ Large protected areas · Biological diversity $\cdot$ Evaluation · Indicators

\section{Einleitung und Problemstellung}

Seit der Mitte des 20. Jahrhunderts besitzen anthropogen genutzte Flächen weltweit einen höheren Anteil als natürliche Landschaften (Ellis/Ramankutty 2008). Auch weiterhin gehen natürliche Ökosysteme in ihrem Bestand stark zurück (MEA 2005), während die Nutzungsintensität der Kulturökosysteme steigt (Stoate/Boatman/Borralho et al. 2001; Lindenmayer/Cunningham/Young 2012). Diese Entwicklung ist eine der Hauptursachen für den globalen Verlust der biologischen Vielfalt (Pereira/Navarro/Martins 2012). Allerdings sind die Auswirkungen der land- und forstwirtschaftlichen Nutzung stark von ihrer Art und Intensität abhängig. So gibt es auf der einen Seite zahlreiche Beispiele für Landnutzungssysteme, die ein hohes Niveau an biologischer Vielfalt gewährleisten (Reidsma/ Tekelenburg/van den Berg et al. 2006). Viele Arten und Biotope Mitteleuropas sind in ihrem Bestand auf eine extensiv genutzte Kulturlandschaft angewiesen (Meyer/ Wesche/Krause et al. 2014). Auf der anderen Seite ging jedoch mit steigender Intensivierung und Homogenisierung der landwirtschaftlichen Nutzung ein anhaltender Verlust biologischer Vielfalt einher (Wesche/Krause/Culmsee et al. 2012; Haber 2014). Demgegenüber sind die Auswirkungen der forstwirtschaftlichen Nutzung auf die biologische Vielfalt in Deutschland weniger eindeutig und werden entsprechend kontrovers diskutiert (Meyer 2013; Bässler/ Müller 2015; Bolte 2015; Bork/Knoke 2015).

Mit Unterzeichnung der Konvention zur biologischen Vielfalt (Convention on Biological Diversity (CBD)) hat sich Deutschland 1993 völkerrechtlich dazu verpflichtet, die biologische Vielfalt zu erhalten, wiederherzustellen und ihre nachhaltige und faire Nutzung sicherzustellen. Diese Verpflichtung kann sowohl ethisch als auch funktional (Hooper/Chapin/Ewel et al. 2005; Cardinale/Duffy/ Gonzalez et al. 2012) gut begründet werden. Dabei bezieht sich die Erhaltungsverpflichtung auf die naturraumtypische biologische Vielfalt, um eine globale Homogenisierung $\mathrm{zu}$ vermeiden (Haber 2004; Lindenmayer/Hunter 2010). Die Erhaltung der biologischen Vielfalt und der Nutzungsfähigkeit der Naturgüter sind auch vorrangige Ziele des Bundesnaturschutzgesetzes (Fassung vom 29. Juli 2009) sowie als zentraler Baustein einer nachhaltigen Entwicklung seit 1995 Staatsziel im deutschen Grundgesetz (Artikel 20a).

Die land- und forstwirtschaftliche Nutzung steht aufgrund ihrer Versorgungsleistungen und ihrer Bedeutung für die Bewahrung der biologischen Vielfalt vor großen Herausforderungen. Sie ist nicht nur aufgerufen, Nahrungsmittel und nachwachsende Rohstoffe in vermutlich steigender Menge nachhaltig zu produzieren, sondern auch die Belange des Naturschutzes in stärkerem Maße als bisher zu berücksichtigen. Nur durch einen signifikanten Beitrag von Land- und Forstwirtschaft ist es möglich, den akuten Verlust der biologischen Vielfalt aufzuhalten. In diesem $\mathrm{Zu}$ sammenhang kann Schutzgebieten eine wichtige Rolle in der Raumordnung zukommen (Job 2010). Sie können Modellräume für eine Integration von Landnutzung und Naturschutz darstellen oder als Vorrangflächen des Naturschutzes Konflikte auf der lokalen bis regionalen Ebene entzerren, um Nutzungs- und Schutzziele auf einer höheren räumlichen Ebene $\mathrm{zu}$ integrieren.

Vor diesem Hintergrund untersuchen wir im vorliegenden Beitrag die folgenden Fragestellungen:

- Was kennzeichnet einen nachhaltigen Umgang der Landund Forstwirtschaft mit der biologischen Vielfalt?

- Wie nachhaltig ist die gegenwärtige Landnutzung im Hinblick auf die biologische Vielfalt?

- Welchen Beitrag leisten die deutschen Großschutzgebiete zur Verwirklichung einer nachhaltigen land- und forstwirtschaftlichen Nutzung?

\section{Nachhaltige Landnutzung}

\subsection{Begriffsbestimmung von Landnutzung und Naturschutz}

Unter Landnutzung ist die Beanspruchung und Bewirtschaftung von Landflächen einschließlich ihrer natürlichen Ressourcen zur menschlichen Bedürfnisbefriedigung $\mathrm{zu}$ verstehen. Dies schließt a) die unmittelbare Entnahme von Rohstoffen aus der Natur, b) die Umgestaltung und Bewirtschaftung von Ökosystemen zur Erzeugung von Nahrungsmitteln und nachwachsenden Rohstoffen sowie c) die 
Nutzung für die Erholung, Umweltbildung oder Forschung ein. Dieser Beitrag beschränkt sich auf die land- und forstwirtschaftliche Nutzung und damit auf den Aspekt b) dieser umfassenderen Definition. Unter Naturschutz verstehen wir ein Element von Landnutzungssystemen, das Nutzungsart und -intensität mit Rücksicht auf die biologische Vielfalt abwandelt, einschränkt oder auf Teilflächen vollständig ausschließen kann. Wir behandeln aus Gründen des Umfangs nicht den Teilbereich Umweltschutz, das heißt den Schutz abiotischer Ressourcen, der nach dem Bundesnaturschutzgesetz in der Fassung von 2009 ebenfalls zum Naturschutz gehört.

\subsection{Unterschiede und Gemeinsamkeiten land- und forstwirtschaftlicher Nutzung}

Gemeinsam ist Land- und Forstwirtschaft, dass sie unmittelbar auf die Produktionsleistungen biologischer Systeme angewiesen sind. Vor allem im Zuge der landwirtschaftlichen Nutzung wurden die ursprünglichen Ökosysteme und die genetische Ausstattung der Tier- und Pflanzenarten stark verändert, um eine möglichst störungsfreie und den menschlichen Bedürfnissen angepasste Produktion in hoher Qualität und Menge zu gewährleisten. Dieses Ziel wurde zwar mit der modernen Landwirtschaft erreicht, die damit verbundene Homogenisierung der Nutzungssysteme und die hohe Stoff- und Energiezufuhr haben aber nicht nur zu einer Belastung der Umwelt, sondern auch zu einer erheblichen Gefährdung der biologischen Vielfalt geführt (Haber 2014). Bei Fortsetzung des gegenwärtigen Trends muss befürchtet werden, dass hierdurch die ökologischen Produktionsgrundlagen der Landwirtschaft sowie andere Ökosystemleistungen signifikant beeinträchtigt werden.

Land- und Forstwirtschaft unterscheiden sich grundlegend in der Dauer ihrer Produktionszyklen und der Naturnähe ihrer Nutzungssysteme (Spellmann 2013). Die meisten Agrarökosysteme, wie Ackerflächen oder Saatgrünland, weisen nur wenige Analogien zu natürlichen Systemen auf, sind in ihrem Bestand auf anthropogene Energie- und Stoffzufuhr angewiesen und unterliegen in der Regel einem jährlichen Nutzungszyklus. Hingegen weisen die meisten Wirtschaftswälder vielfach Strukturen und Prozesse auf, die bei allen Unterschieden in Art, Menge und Intensität - ana$\log$ auch in natürlichen Wäldern beobachtet werden können. Zudem werden Wirtschaftswälder zumindest in Mitteleuropa überwiegend in einem viele Jahrzehnte dauernden Produktionszeitraum bewirtschaftet. Die Möglichkeiten der Steuerung der Produktionsbedingungen und der technische Fortschritt sind in der Landwirtschaft ungleich größer als in der Forstwirtschaft. Gleichzeitig unterliegt der landwirtschaftliche Sektor weitaus stärker den Witterungseinflüssen und der Volatilität von Märkten und Preisen.

\subsection{Was ist nachhaltige land- und forstwirtschaftliche Nutzung?}

Nachhaltige land- und forstwirtschaftliche Nutzung kann im Sinne der Definition der Weltkommission für Umwelt und Entwicklung (WCED 1987) als eine Art und Weise der Bewirtschaftung von Landflächen aufgefasst werden, bei der die Bedarfsdeckung der heutigen Generation diejenige der kommenden Generationen nicht einschränkt. Dies setzt voraus, dass das Nutzungspotenzial der bewirtschafteten Systeme dauerhaft aufrechterhalten oder verbessert wird. Das Nutzungspotenzial wird heute üblicherweise nach dem Drei-Säulen-Prinzip in eine soziale, eine ökonomische und eine ökologische Dimension unterteilt (Deutscher Bundestag 1998). Damit erhält Nachhaltigkeit neben der zeitlichen eine funktionale Dimension. Allerdings erwachsen grundlegende Bewertungsprobleme daraus, dass sowohl die zukünftige Ökosystementwicklung als auch die Bedürfnisse künftiger Generationen nicht ausreichend sicher abgeschätzt werden können (Lamprecht 1970; Haber 2014). Der Abwägungsprozess zwischen Gegenwartsund Zukunftsnutzen erfordert kontext- und zeitspezifische Lösungen (Schanz 1995). Für eine nachhaltige Landund Forstwirtschaft gibt es daher keine operationale und zugleich allgemein anerkannte Definition (Walter/Stützel 2009: 1276). Dies zeigt beispielsweise auch die Vielfalt des Nachhaltigkeitsverständnisses im Rahmen des Förderschwerpunktes „Nachhaltiges Landmanagement“ des Bundesministeriums für Bildung und Forschung (BMBF) (vgl. Weith/Besendörfer/Gaasch et al. 2013).

\subsection{Funktionierende Managementsysteme als Kennzeichen nachhaltiger Land- und Forstwirtschaft}

Nachhaltigkeit der Landnutzung kann als Annäherungsprozess an die Leitidee generationenübergreifender Gerechtigkeit aufgefasst werden. Daher ist ein funktionierendes, auf die Gewährleistung von dauerhafter Multifunktionalität ausgerichtetes Managementsystem eines der wichtigsten Kennzeichen von Nachhaltigkeit. Das Managementsystem sollte geeignet sein, die Wirtschaftsweise den sozialen, ökonomischen und ökologischen Zielen regelmäßig anzupassen und damit die Landnutzung nachhaltig auszurichten. Ein solches Managementsystem für die Landnutzung stellt ein sozio-ökologisches System mit verschiedenen Sub-Systemen dar (Ostrom 2009):

- Ressourcensystem: die räumliche Nachhaltigkeitseinheit, z.B. die landwirtschaftliche Nutzfläche einer bestimmten Region oder die in einem Schutzgebiet befindliche Fläche 
Abb. 1 Idealtypischer Regelkreis der Bewirtschaftung innerhalb des Nutzersystems und die wichtigsten Querbeziehungen zu anderen Sub-Systemen der Landnutzung

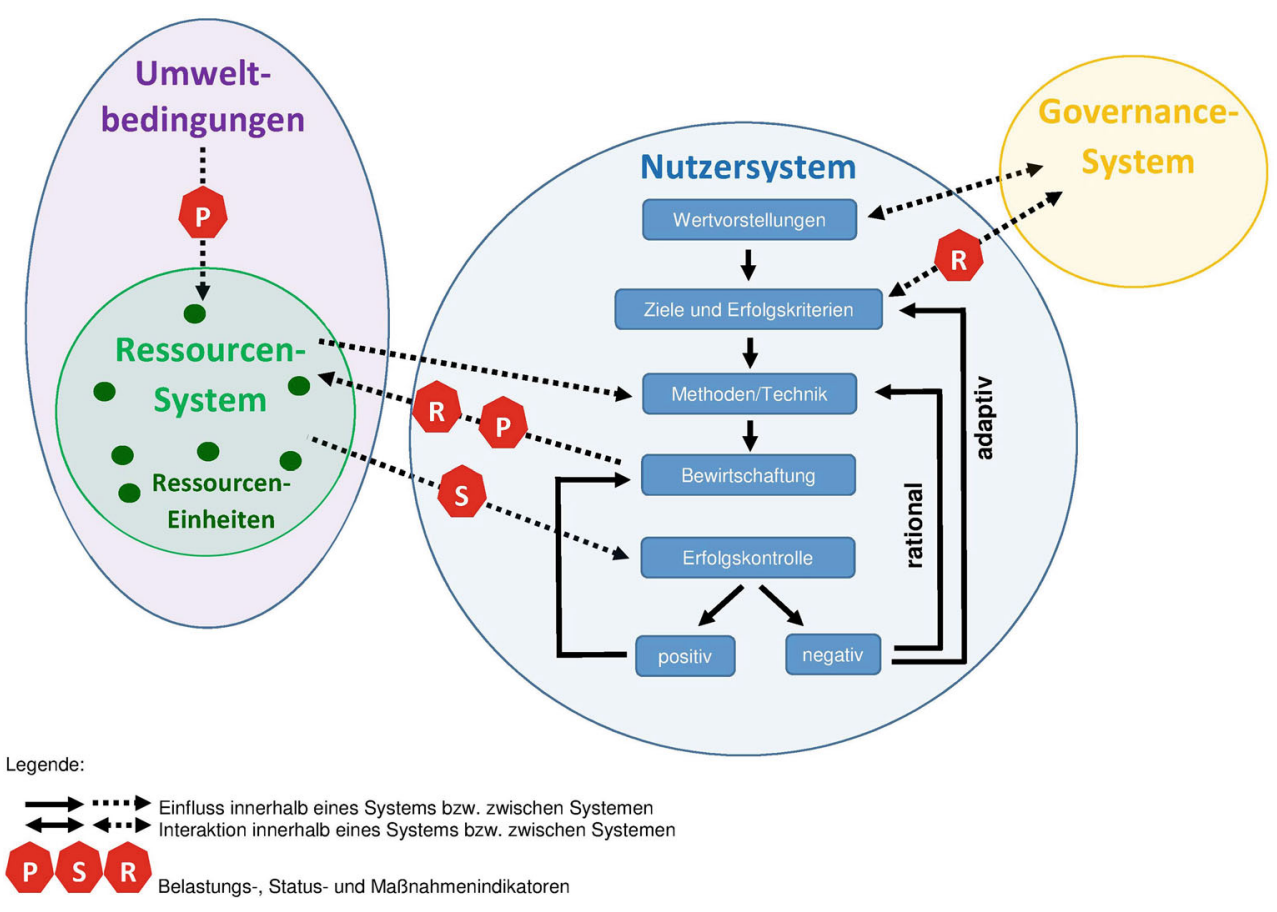

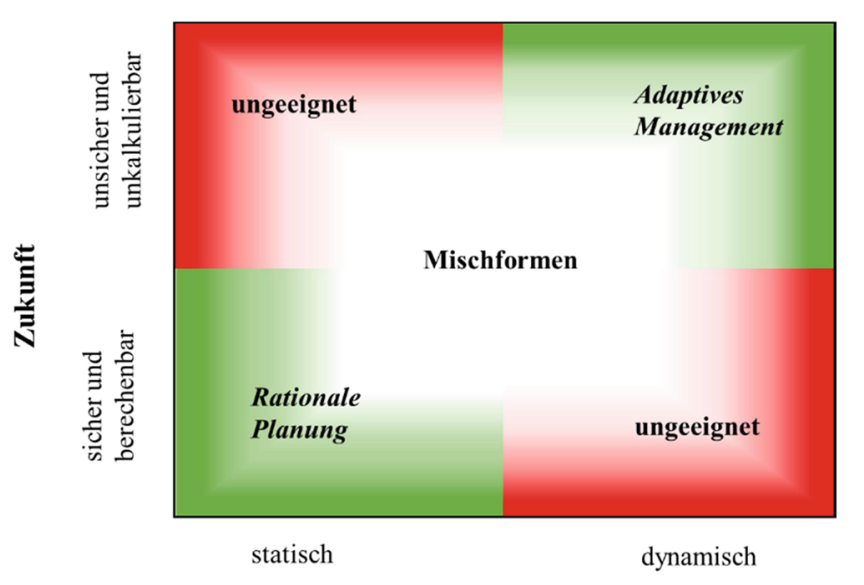

Ziele und Maßnahmen

Abb. 2 Eignung rationaler bzw. adaptiver Planung in Abhängigkeit vom Grad der Unsicherheit zukünftiger Entwicklungen

- System der Ressourcenelemente: die Summe der bewirtschafteten und/oder geschützten Ressourcen, wie z.B. Acker- und Grünlandflächen, Wildtiere, Waldbestände, das Grundwasser und deren Beziehungen untereinander

- Governance-System: Regierungen und andere Organisationen, die das Ressourcensystem managen einschließlich ihrer Regelwerke

- Nutzersystem: Die Gruppe(n), die das Ressourcensystem auf unterschiedliche Art und Weise nutzen, z. B. zur erwerbswirtschaftlichen Rohstoffproduktion oder auch zur Erholung
Diese Sub-Systeme sind zum Teil unterschiedlichen Maßstabsebenen zuzuordnen, sodass ihre Interaktionen auch skalenübergreifend betrachtet werden müssen (Becken/Job 2014). Das Nutzersystem wird sinnvollerweise in Form eines Regelkreises organisiert, der in einem angemessenen zeitlichen Turnus durchlaufen werden sollte, um die Zielerreichung zu messen und gegebenenfalls Anpassungsmaßnahmen vorzunehmen (vgl. Abb. 1).

Je nach Sicherheit oder Unsicherheit der Zukunft ist entweder eine rationale oder adaptive Ausrichtung des Managementsystems sinnvoll (vgl. Abb. 2). Im Unterschied $\mathrm{zu}$ einer rationalen Planung unterliegen bei einer adaptiven Vorgehensweise auch die Zielsetzungen dem Anpassungsprozess (Hoogstra-Klein/Burger 2013). Dies geht mit der Gefahr einher, dass sinnvolle Ziele aufgegeben werden, weil ihre Erreichung Schwierigkeiten macht. Im Ergebnis würden die Zielwerte nach unten verschoben werden (,Shifting-Baseline-Syndrom“; vgl. Pauly 1995). Hingegen sind ausschließlich rationale Managementsysteme zu starr, um auf veränderte Anforderungen und Rahmenbedingungen reagieren zu können, was insbesondere bei den hohen Unsicherheiten einer sich stark verändernden Welt sehr kritisch ist (Ulrich 1991; Puettmann/Messier/Coates 2014).

\subsection{Indikatorensysteme zur Bewertung der Nachhaltigkeit der Land- und Forstwirtschaft im Hinblick auf die biologische Vielfalt}

Angesichts der heutigen Komplexität der Ziele und Wirkungszusammenhänge auf unterschiedlichen räumlichen und zeitlichen Skalen kann Nachhaltigkeit nur über ein 
Tab. 1 Ergebnisse von Indikatorensystemen zur Bewertung der Nachhaltigkeit der land- und forstwirtschaftlichen Nutzung im Hinblick auf die biologische Vielfalt. NBS Nationale Strategie zur Biologischen Vielfalt, SEBI Streamlining European Biodiversity Indicators, $D L G$ Deutsche Landwirtschaftliche Gesellschaft, MCPFE Ministerial Conference on the Protection of Forests in Europe Typ: Belastungs$(P)$, Zustands- $(S)$, MaßnahmenIndikator $(R)$

\begin{tabular}{|c|c|c|c|c|c|c|}
\hline \multicolumn{2}{|c|}{ Ebene der biologischen Vielfalt / Indikator } & \multirow{2}{*}{$\begin{array}{c}\text { Typ } \\
P\end{array}$} & \multirow{2}{*}{ NBS } & \multirow{2}{*}{$\begin{array}{c}\text { SEBI } \\
-\end{array}$} & \multirow{2}{*}{$\frac{\text { DLG }}{-}$} & \multirow{2}{*}{$\frac{\text { MCPFE }}{-}$} \\
\hline$\frac{g}{0}=$ & Gentechnik in der Landwirtschaft [ha] & & & & & \\
\hline 急 焉 & Genetische Vielfalt Landwirtschaft [\%] & $\mathrm{s}$ & 0 & O & - & - \\
\hline 5 & Genetische Ressourcen Forstwirtschaft [\%] & s & - & - & - & $\nearrow$ \\
\hline \multirow{10}{*}{ 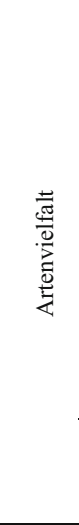 } & Gefährdete Arten ${ }^{\mathrm{a}}$, & $\mathrm{S}$ & $\searrow$ & $(\searrow)$ & & 0 \\
\hline & Kulturpflanzendiversität [\%] & $\mathrm{S}$ & - & - & 0 & - \\
\hline & Artenvielfalt und Landschaftsqualität [\%] ${ }^{\mathrm{b}}$ & $\mathrm{S}$ & $\searrow$ & - & - & - \\
\hline & Teilindikator „Agrarland“ [\%] & $\mathrm{s}$ & $\downarrow$ & - & $\downarrow$ & - \\
\hline & Teilindikator „Wälder“ [\%] & $\mathrm{S}$ & ○ & - & - & - \\
\hline & Vorkommen/Verteilung Feldvögel/Grünlandschmetterlinge [\%] & S & - & $(\downarrow)$ & - & - \\
\hline & Vorkommen/Verteilung Waldvögel [\%] & $\mathrm{S}$ & - & $(\searrow)$ & - & - \\
\hline & Waldbaumartenzusammensetzung & $\mathrm{S}$ & - & - & - & 0 \\
\hline & Fremdländische Baumarten [\%] & $P$ & - & - & - & 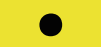 \\
\hline & Erhaltungszustand FFH-Lebensraumtypen und -arten $[\%]^{\mathrm{c}}$ & $\mathrm{S}$ & $\searrow$ & $(\bigcirc)$ & - & - \\
\hline \multirow{10}{*}{ 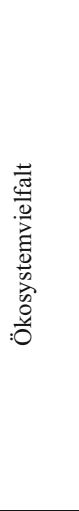 } & Eutrophierende Stickstoffeinträge [\%] & $P$ & $\nearrow$ & $(\nearrow)$ & - & 0 \\
\hline & Stickstoffüberschussreduzierung Landwirtschaft $\left[\mathrm{kg} \mathrm{ha} \mathrm{a}^{-1}\right]^{\mathrm{d}}$ & $\mathrm{P}$ & $\nearrow$ & $\nearrow$ & $\nearrow$ & - \\
\hline & Landwirtschaftsflächen mit hohem Naturwert [\%] ${ }^{\mathrm{e}}$ & $S(R)$ & $\searrow$ & 0 & - & - \\
\hline & Ökologischer Landbau [\%] & $\mathrm{R}$ & $\uparrow$ & $\uparrow$ & - & - \\
\hline & Totholz $\left[\mathrm{m}^{3} / \mathrm{ha}\right]$ & $\mathrm{R}(\mathrm{S})$ & - & $\nearrow$ & - & $\nearrow$ \\
\hline & Anteil Waldflächen mit natürlicher Verjüngung [\%] & $\mathrm{R}$ & - & - & - & $\nearrow$ \\
\hline & Naturnähe der Wälder [\%] & $\mathrm{S}$ & - & - & - & 0 \\
\hline & Nachhaltige Forstwirtschaft [\%] & $\mathrm{R}$ & $\nearrow$ & - & - & - \\
\hline & Agrarumweltmaßnahmen & $\mathrm{R}$ & $\nearrow$ & - & - & - \\
\hline & Schutzgebietsflächen/Gebietsschutz [\% ${ }^{\mathrm{f}}$ & $\mathrm{R}$ & $\nearrow$ & $(\nearrow)$ & - & $\uparrow$ \\
\hline \multirow{3}{*}{ 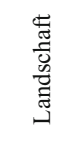 } & Flächeninanspruchnahme für Siedlung und Verkehr & $P$ & $\uparrow$ & - & $\nearrow$ & - \\
\hline & Landschaftszerschneidung [\%] & $\mathrm{P}$ & $\searrow$ & - & - & - \\
\hline & Landschaftsmosaik Waldfläche & $\mathrm{S}$ & - & - & - & $\nearrow$ \\
\hline
\end{tabular}

Zustandsbewertung: grün = positiv, gelb $=$ bedingt positiv, rot $=$ negativ, grau $=$ keine Bewertung

Trendaussagen: $\uparrow=$ stark positiver Trend, $\downarrow=$ stark negativer Trend, $\lambda=$ bedingt positiver Trend, $\downarrow=$ bedingt negativer

Trend, $\mathbf{O}=$ keine Veränderung, $\bigcirc=$ keine Trendaussage; in Klammern = nur europaweite Aussage

a bei MCPFE = nur gefährdete Waldbaumarten, bei SEBI = Rote Liste Index gefährdeter europäischer Vogelarten, bei NBS $=$ Rote Liste Kategorien für ausgewählte Artengruppen (zurzeit Wirbeltiere, Flechten, Schleimpilze, Großalgen, 28 Gruppen der Wirbellosen)

${ }^{\mathrm{b}}$ bei SEBI $=$ Häufigkeit und Verteilung von ausgewählten Arten, bei DLG = Biodiversität

$c$ bei SEBI $=$ Arten und Habitate von europäischem Interesse

d bei DLG $=$ andere Berechnungsgrundlage als bei NBS und SEBI, daher andere Bewertung

e bei NBS und SEBI $=$ High Nature Value Farmland (HNV-Index)

f bei NBS = Anteil an Naturschutzgebieten, Nationalparks sowie ausgewiesenen Kern- und Pflegezonen der Biosphärenreservate, bei SEBI = Anteil geschützter Gebiete und Natura2000-Flächen, bei MCPFE $=$ Waldschutzgebiete nach MCPFE Klassen 1.1-1.3 und 2
System aussagekräftiger, die relevanten Bereiche abdeckende Indikatoren und deren Monitoring ermittelt werden (Hurni 2000). Indikatoren fassen komplexe Sachverhalte verständlich zusammen, um die nicht unmittelbar erkennbaren Zustände und Veränderungen sichtbar zu machen (Pirk1/Riedel 1991: 343). Sie können einzeln oder auch als Teil eines Indikatorensystems genutzt werden (Dantsis/ Douma/Giourga et al. 2010). Zur Bewertung von Status quo und Entwicklungsrichtung des betrachteten Parameters ist die Festlegung von Zielwerten erforderlich.

Um den Regelkreis eines Nutzersystems (vgl. Abb. 1) zu verwirklichen, sollten die Indikatoren so kombiniert werden, dass Aussagen über Belastungsfaktoren mit Zustandsveränderungen in Verbindung gebracht werden können. Ebenso sollten die Wirkungen der ergriffenen Maßnahmen in Form von Zustandsänderungen der Zielgrößen erkennbar 

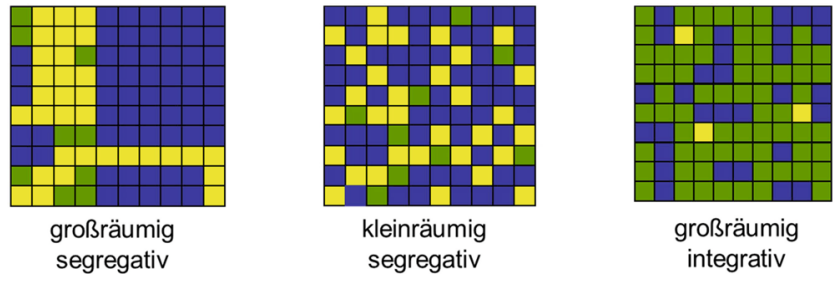

Abb. 3 Grundmuster der räumlichen Differenzierung der Landnutzung. Gelb: Vorrangflächen Naturschutz, blau: Vorrangflächen landoder forstwirtschaftliche Produktion, grün: Integration von Naturschutz und Produktion auf gleicher Fläche. Die Anteile der gelben und blauen Kästchen sind in jedem Raummuster gleich, wobei durch Integration von Naturschutz und Produktion auf der gleichen Fläche ein grünes Kästchen entsteht

sein. Die einfachste Form eines solchen kausal angelegten Indikatorensystems besteht daher aus Belastungs-, Zustands- und Maßnahmenindikatoren (Hayati/Ranjbar/ Karami 2010), die auch als PSR-Indikatoren (Pressure, State, Response) bezeichnet werden. ${ }^{1}$

Indikatorensysteme zur Bewertung der Nachhaltigkeit forst- und landwirtschaftlicher Nutzung wurden im europäischen Raum beschleunigt seit den 1990er-Jahren im Nachgang zur Agenda 21 entwickelt. Das erste Indikatorensystem waren die bis heute fortgeführten „Pan-European Indicators for Sustainable Forest Management" der Ministerkonferenz zum Schutz der Wälder in Europa (vgl. Schneider 1995; Spellmann 2003). Mittlerweile liegen für die meisten Indikatoren länderweise Werte von 1990 bis 2010 vor (vgl. Forest Europe Liaison Unit Oslo 2011). Das Kriterium „Erhaltung, Schutz und angemessene Verbesserung der biologischen Diversität in Forstökosystemen“ (biologische Diversität) wird anhand von zehn Indikatoren bewertet (vgl. Tab. 1). Für den Agrarbereich wurde im Jahr 2008 der DLGNachhaltigkeitsstandard für die Landwirtschaft entwickelt (Christen/Hövelmann/Hülsbergen et al. 2009). Die biologische Vielfalt wird mit vier Indikatoren abgebildet (vgl. Tab. 1), deren Verlauf für unterschiedlich lange Zeiträume dokumentiert ist (DLG 2015).

Neben den multifunktional angelegten Indikatorensystemen der Land- und Forstwirtschaft geben auch die naturschutzfachlichen Indikatorensysteme Auskunft über die Nachhaltigkeit der Landnutzung im Hinblick auf die biologische Vielfalt. Zwischen den Systemen gibt es zudem Schnittmengen, da teilweise identische oder ähnliche Indikatoren in mehreren Systemen verwendet werden. Auf europäischer Ebene wurde 2005 von der Europäischen Umweltagentur (EEA) das SEBI-Indikatorensystem (,Streamlining European Biodiversity Indicators") entwickelt. Das System besteht aus insgesamt 26 Indikatoren, die jedoch nicht alle nach Ländern aufgeschlüsselt sind.

1 Vgl. http://ia2dec.pbe.eea.europa.eu/knowledge_base/Frameworks/ doc101182 (14.07.2016).
Die Nachhaltigkeit der land- und forstwirtschaftlichen Nutzung im Hinblick auf die biologische Vielfalt wurde in der vorliegenden Untersuchung auf der Grundlage der derzeit auf der nationalen Ebene eingeführten Indikatorensysteme bewertet (vgl. Tab. 1). Dabei handelt es sich um die Indikatorensysteme der ,Nationalen Strategie zur Biologischen Vielfalt“ (NBS) (BMUB 2015a), des SEBI-Systems (EEA 2015), des Systems der Deutschen Landwirtschaftlichen Gesellschaft (DLG 2015) und der „Ministerial Conference on the Protection of Forests in Europe“ (MCPFE) (Forest Europe Liaison Unit Oslo 2011). Die einzelnen Indikatoren wurden den verschiedenen Ebenen der biologischen Vielfalt (genetische Vielfalt, Arten-, Ökosystem- und Landschaftsvielfalt) zugeordnet und nach Belastungs-, Zustands- oder Maßnahmenindikatoren typisiert. Anschließend wurden Zustand und Trend nach einer Ordinalskala bewertet.

Für alle Ebenen der biologischen Vielfalt liegen Indikatoren vor. Allerdings weisen die einzelnen Systeme erhebliche Unterschiede auf. Ein Zusammenhang zwischen Belastungs- bzw. Maßnahmenindikatoren und ihren Wirkungen kann für keinen Themenbereich unmittelbar hergestellt werden (z. B. Eutrophierung und damit zusammenhängende Diversitätsverluste). Hinzu kommt, dass für einige Indikatoren keine Zielwerte formuliert wurden (in Tab. 1 weiß bzw. grau hinterlegt), sodass eine Bewertung im eigentlichen Sinne nicht möglich ist. Aussagen zur Arten- und Ökosystemvielfalt sind weitaus differenzierter als Aussagen zur genetischen und Landschaftsvielfalt. In der Gesamtschau zeigen die vorliegenden Ergebnisse eine überwiegend negative Entwicklung der biologischen Vielfalt auf landwirtschaftlichen Flächen. Hingegen ergibt sich für den Wald ein differenzierteres Bild.

\section{Charakteristik und Status quo der Großschutzgebiete in Deutschland}

\subsection{Zielsetzungen der deutschen Großschutzgebiete}

Mit den seit 2005 unter der Dachmarke „Nationale Naturlandschaften" zusammengefassten drei Kategorien von Großschutzgebieten in Deutschland - Naturparke, Biosphärenreservate und Nationalparke - werden unterschiedliche Ziele verfolgt (vgl. Tab. 2). Die Großschutzgebietskategorien sind daher unterschiedlichen Raummustern der Landnutzung (vgl. Abb. 3) zuzuordnen. Da in Nationalparken in erster Linie eine vom Menschen nicht aktiv beeinflusste Entwicklung geschützt wird, schließen sich hier land- und forstwirtschaftliche Nutzungen auf der überwiegenden Fläche aus. Nationalparke stellen großräumig-segregative Elemente der Raumordnung dar. Als Modellgebiete nachhaltiger Landnutzung (vgl. Gehrlein/Kullmann 2011) verfol- 
Tab. 2 Ziele der drei Großschutzgebietskategorien in Deutschland (nach Bundesnaturschutzgesetz in der Fassung vom 29. Juli 2009) und damit verbundene Restriktionen für die Landnutzung

\begin{tabular}{|c|c|c|c|c|c|c|c|}
\hline \multirow{2}{*}{ Großschutzgebiet } & \multicolumn{3}{|c|}{$\begin{array}{l}\text { Schutzregime zur Erhaltung der } \\
\text { biologischen Vielfalt }\end{array}$} & \multirow{2}{*}{ 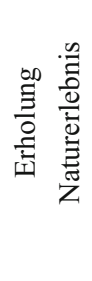 } & \multirow{2}{*}{ 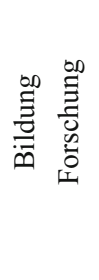 } & \multirow{2}{*}{ 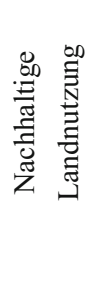 } & \multirow{2}{*}{ 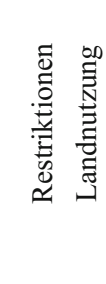 } \\
\hline & $\begin{array}{l}\text { Eigen- } \\
\text { dynamik }\end{array}$ & $\begin{array}{c}\text { Pflege } \\
\text { und } \\
\text { Nutzung }\end{array}$ & $\begin{array}{l}\text { Vielfalt } \\
\text { der } \\
\text { Land- } \\
\text { schaft }\end{array}$ & & & & \\
\hline Nationalpark & 0 & 0 & 0 & 0 & $\odot$ & 0 & 0 \\
\hline Biosphärenreservat & $\bigcirc$ & 0 & 0 & 0 & $\odot$ & 0 & $\bigcirc-(\mathbf{O})$ \\
\hline Naturpark & O & 0 & 0 & 0 & $\bigcirc$ & 0 & O \\
\hline
\end{tabular}

gen Biosphärenreservate eher einen kleinräumig-segregativen bis großräumig-integrativen Ansatz. Noch stärker in Richtung eines großräumig-integrativen Musters sind Naturparke einzuordnen. Sie dienen der Erhaltung, Entwicklung oder Wiederherstellung einer durch vielfältige Nutzungen geprägten Landschaft. In ihnen soll eine dauerhaft umweltgerechte Landnutzung verwirklicht werden (vgl. Job 2016).

\subsection{Beitrag der Großschutzgebiete zur Entwicklung einer nachhaltigen land- und forstwirtschaftlichen Nutzung}

\subsubsection{Räumliche Verteilung und Repräsentativität der deutschen Großschutzgebiete}

Die drei Großschutzgebietskategorien nehmen sehr unterschiedliche Anteile der Landfläche Deutschlands ein (vgl. Abb. 4). Während die derzeit bestehenden 16 Nationalparke $0,6 \%$ der terrestrischen Fläche umfassen, liegen die Anteile der 17 Biosphärenreservate bei 3,7\% und der 105 Naturparke bei 27,2\% (Job 2016).

Je nach Großschutzgebietskategorie lassen sich unterschiedliche Verteilungsmuster auf die Großlandschaften erkennen (vgl. Abb. 5). Mit Nationalparken werden die Alpen, die ost- und westdeutschen Mittelgebirge und das nordostdeutsche Tiefland gut repräsentiert. Hingegen sind das Alpenvorland, die südwestdeutschen Schichtstufenlandschaften und das nordwestdeutsche Tiefland deutlich unterproportional vertreten. Biosphärenreservate besitzen im Alpenvorland, in den ostdeutschen Mittelgebirgen und im nordwestdeutschen Tiefland nur geringe Flächenanteile. Naturparke sind bis auf die Alpen und das Alpenvorland in allen Großlandschaften mit größeren Anteilen vertreten.

\subsubsection{Hauptergebnisse der Evaluierung deutscher Großschutzgebiete}

In den letzten Jahren wurden die Leistungen und Defizite der Großschutzgebiete in Deutschland mehrfach bewertet (Schrader 2006; Schrader 2010; BfN 2010; DRL 2010; Heiland/Hoffmann 2013; Schumacher/Job 2013). Dadurch kann in etwa abgeschätzt werden, wie gut sie die ihnen zugedachten Aufgaben erfüllen.

Nach Einschätzung des Bundesamtes für Naturschutz (BfN 2010) bilden Nationalparke und Biosphärenreservate die Kernräume des Biodiversitätsschutzes in Deutschland. Großschutzgebiete leisten demnach einen wichtigen Beitrag zum Ziel der Wildnisentwicklung in der ,Nationalen Strategie zur biologischen Vielfalt" (NBS) und zur positiven Populationsentwicklung wichtiger Schlüsselarten (BfN 2010: 8). Das Bundesamt für Naturschutz (BfN 2010: 16) geht davon aus, dass Initiativen zur nachhaltigen Entwicklung vor allem in Biosphärenreservaten und Naturparken entstanden sind. Als Handlungserfordernisse sieht das Bundesamt in den meisten Naturparken eine deutliche Erhöhung des Stellenwerts des Naturschutzes und die verstärkte Umstellung auf naturverträgliche Nutzungen (vgl. BfN 2010). Großschutzgebiete sollten zudem ihrer Vorbildfunktion im Hinblick auf eine Minderung der Freiflächeninanspruchnahme für Siedlungen und Verkehr besser gerecht werden. Sowohl innerhalb der Großschutzgebiete als auch zwischen den Großschutzgebieten sollte der Biotopverbund verstärkt gefördert werden. Schließlich sollen zur Überprüfung der Wirkung und Optimierung der durchgeführten Maßnahmen Erfolgskontrollen durchgeführt werden.

Diese Bewertung der Leistungen der Großschutzgebiete beruht nicht auf Vergleichsdaten zu anderen Schutzgebieten und der nicht geschützten Landschaft. Inwieweit die positiven Entwicklungen direkt den Großschutzgebieten zuzurechnen sind, bleibt daher fraglich. Auch die Handlungsfelder zur Weiterentwicklung der Großschutzgebiete basieren nicht durchgehend auf einer transparenten Evaluierung. 
Abb. 4 Großschutzgebiete in Deutschland, Stand: 06/2015 (Quelle: Job 2016)

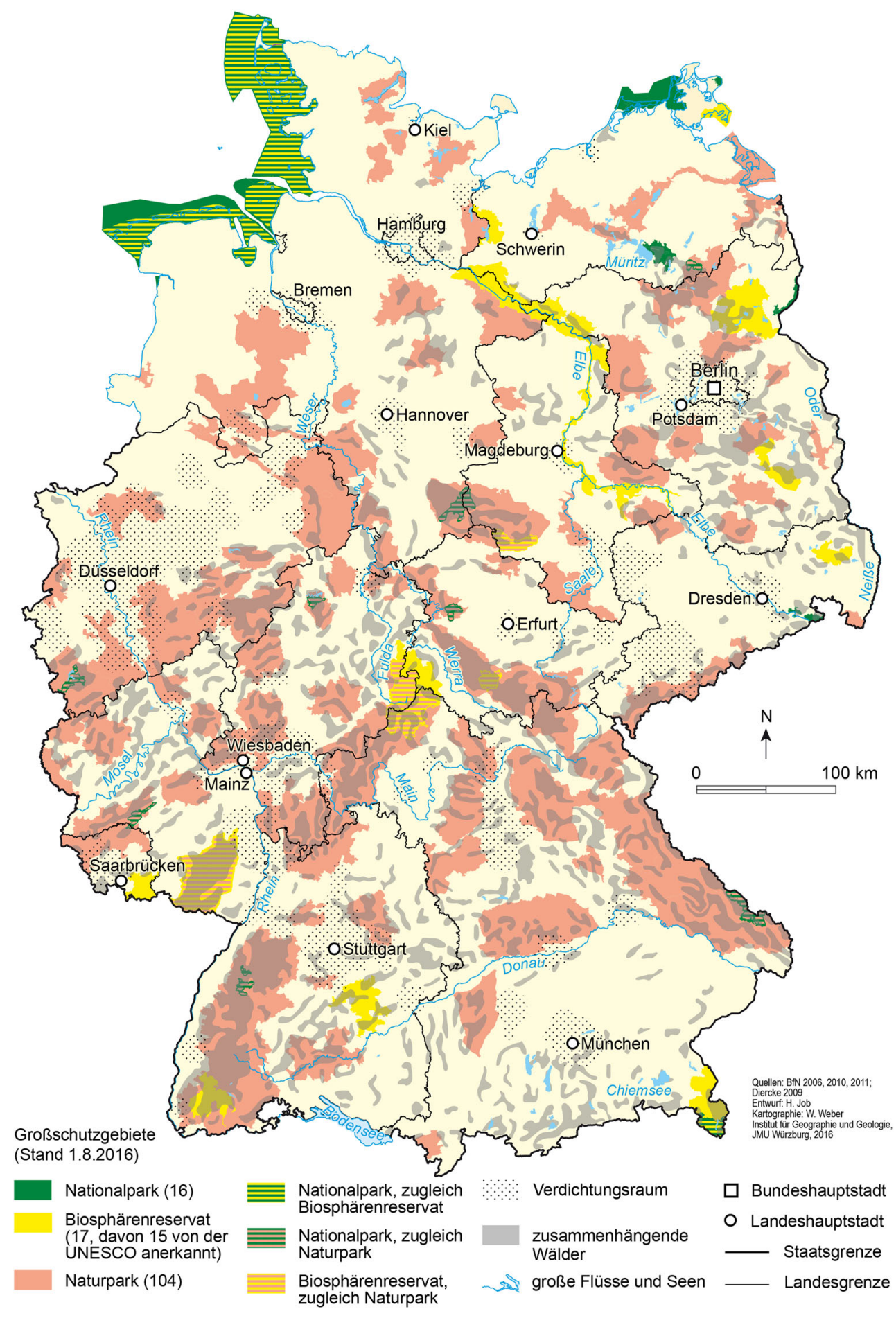

Naturparke sind nach Hager (2009) besonders erfolgreich beim Biotopverbund. Schrader (2006: 56) stellt fest, dass die deutschen Biosphärenreservate vorbildliche Ergebnisse bei der UNESCO-Evaluierung erzielen. Ihre eigene Evaluierung ergab unter anderem die folgenden Hauptergebnisse:
- Die Agrarförderung konterkariert zum Teil die Entwicklung in Richtung einer extensiven landwirtschaftlichen Nutzung.

- Die Biosphärenreservate werden von staatlicher und politischer Seite in vielerlei Hinsicht nicht ausreichend beachtet. Ihre Wahrnehmung in der Öffentlichkeit ist recht 
Abb. 5 Prozentuale Verteilung der terrestrischen Fläche der Großschutzgebietskategorien und Deutschlands auf die Großlandschaften. ALP Alpen, $O M G$ Ostdeutsche Mittelgebirge, $A L V$ Alpenvorland, $W M G$ Westdeutsche Mittelgebirge, $S W G$ Südwestdeutsche Mittelgebirge, NWT Nordwestdeutsches Tiefland, NOT Nordostdeutsches Tiefland (Datenquelle: Bundesamt für Naturschutz, LANIS-BUND, Stand: 2016)

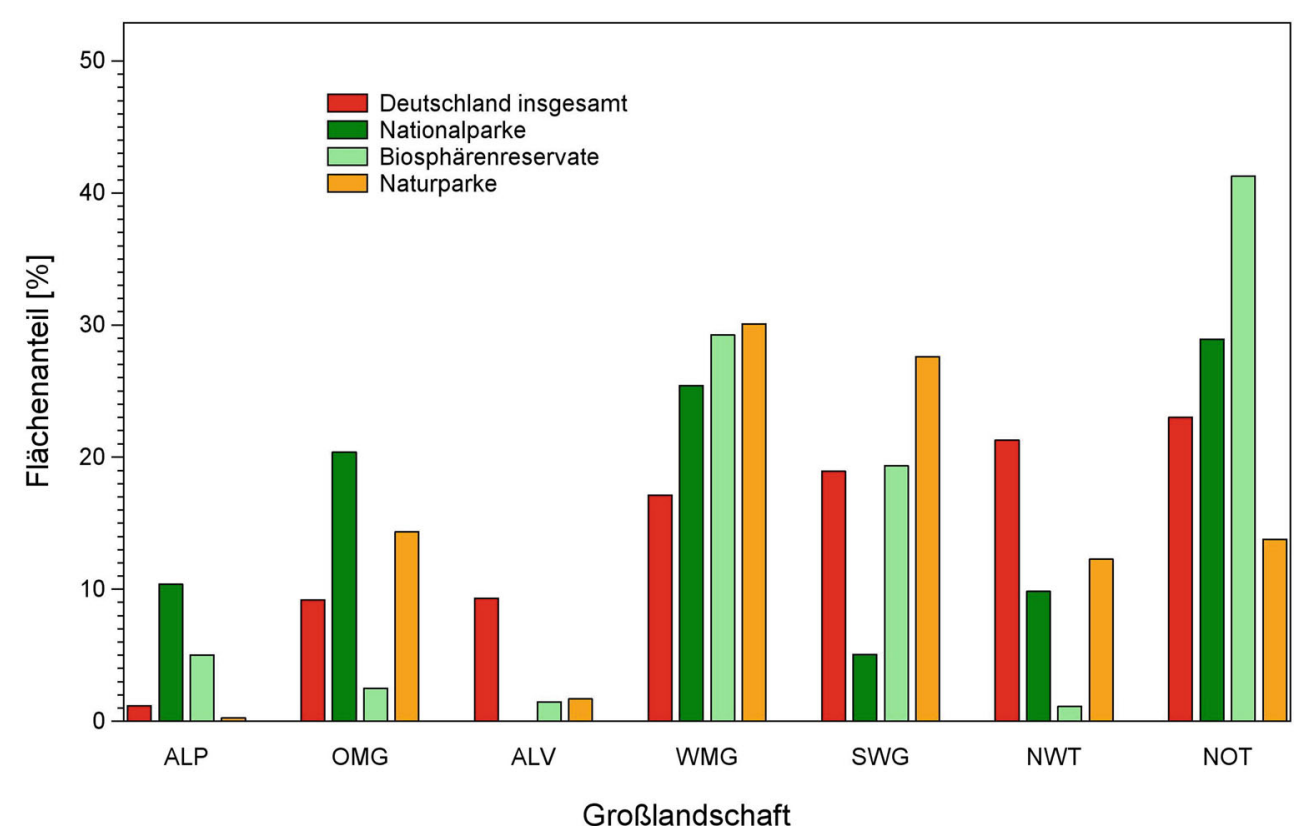

begrenzt und ihre Personal- und Finanzausstattung meist nicht ausreichend.

- Da Biosphärenreservate oft in strukturschwachen Regionen mit einer höheren Arbeitslosigkeit liegen und die lokale Bevölkerung mit der Ausweisung eines Biosphärenreservates strenge Naturschutzauflagen verbindet, ist die Akzeptanz für den Naturschutz gering.

- Es mangelt an einer bundesländerübergreifenden Entwicklung der Biosphärenreservate und der Koordinierung von Fördermöglichkeiten. Weder eine nationale noch eine EU-Förderpolitik sind vorhanden.

- Der Erfolg der Biosphärenreservate ist eng mit dem Engagement einzelner Personen verbunden.

Auch neuere Evaluierungen der Biosphärenreservate Rhön und Südost-Rügen (Jedicke 2013; UNESCO 2013) bestätigen im Wesentlichen diese Einschätzung. Schrader (2006: 662) resümiert, dass Biosphärenreservate als Beispiele eines sinnvollen Miteinanders von Mensch und Natur nur funktionieren können, wenn sie allen sozialen, kulturellen, geistigen und wirtschaftlichen Bedürfnissen der lokalen Bevölkerung gerecht werden und ihr Management auf einer gesicherten wissenschaftlichen Grundlage beruht. So unterliegen beispielsweise die wirtschaftlichen Effekte des Tourismus in den Biosphärenreservaten Deutschlands großen Schwankungen und hängen neben der Größe der Tourismusregion oder der Nähe zu Verdichtungsräumen unter anderem auch vom gemeinsamen Auftreten der touristischen Akteure ab (Job/Kraus/Merlin et al. 2013). Um ihrem Auftrag einer nachhaltigen Entwicklung dabei gerecht zu werden, sollte aus ökonomischer Perspektive das Schließen regionaler Wirtschaftskreisläufe im Vordergrund der Bemühungen stehen (vgl. Kraus/Merlin/Job 2014).
Schumacher/Job (2013) sehen die deutsche Nationalparkgeschichte als Erfolg, identifizieren aber auch Lücken im Nationalparknetz. Sie kritisieren, dass die eigentliche naturschutzfachliche Begründung für Nationalparke bei den Auseinandersetzungen um ihre Einrichtung zugunsten regionalökonomischer Argumente in den Hintergrund tritt. Dabei können die regionalökonomischen Effekte von Nationalparken und von Großschutzgebieten insgesamt beachtlich sein (Job/Harrer/Metzler et al. 2005; Job/Woltering/Harrer 2009). Heiland/Hoffmann (2013) sehen Stärken und Schwächen bei der praktischen Umsetzung der Nationalparkziele und insbesondere bei der Umsetzung eines konsequenten Prozessschutzes. Sie konstatieren deutliche Schwächen bei Forschung, Monitoring und Evaluierung. In Übereinstimmung damit stellen Kowatsch/Hampicke/Kruse-Graumann et al. (2011: 8) für die deutschen Großschutzgebiete insgesamt das Fehlen eines Monitoringsystems fest, mit dem neben der Zielerreichung und Managementeffizienz auch die gesellschaftlichen Effekte der Großschutzgebiete bewertet werden können. In den verschiedenen Evaluierungen der deutschen Großschutzgebiete wird übereinstimmend betont, dass deren Personal- und Mittelausstattung in vielen Fällen nicht für eine befriedigende Aufgabenwahrnehmung ausreicht (Forst/Scherforse 2010; DRL 2010; Heiland/Hoffmann 2013; Scherfose 2016). 


\section{Diskussion}

\subsection{Bewertung der Nachhaltigkeit land- und forstwirtschaftlicher Nutzung}

Die Probleme bei der Bewertung der Nachhaltigkeit landund forstwirtschaftlicher Nutzung beruhen neben der bereits dargestellten Komplexität der Ressourcensysteme und der Unsicherheit der zukünftigen Entwicklung auch darauf, dass die einzelnen Dimensionen von Nachhaltigkeit miteinander in Einklang gebracht werden müssen. Da ökologische, ökonomische und soziale Ansprüche häufig in Konflikt miteinander stehen (Wagner 2004), ist bei bestimmten Nutzungseingriffen eine Minderung anderer Teilfunktionen nahezu unvermeidlich. Solange jedoch die kritischen Grenzen der Erholungsfähigkeit der genutzten Systeme nicht überschritten werden, können die Eingriffe als nachhaltig angesehen werden (Beese 1996: 73). In Bezug auf die Naturschutzfunktion ist demnach eine land- und forstwirtschaftliche Nutzung nachhaltig, wenn sie die Resilienz der biologischen Vielfalt, das heißt ihre Wiederherstellungsfähigkeit (vgl. Holling 1973), nicht überfordert. Dies kann durch ein Managementsystem überprüft werden, welches auf transparenten, messbaren Indikatoren beruht. Eine signifikante Entwicklung in Richtung definierter Zielwerte und die Erreichung eines Zielkorridors zeigen eine nachhaltige Nutzung an. Wichtig ist hierbei die Ableitung gut begründeter Zielwerte bzw. -korridore, um eine Erosion der Funktionenerfüllung nach dem „Shifting-Baseline-Syndrom“ (Papworth/Rist/Coad et al. 2009) zu vermeiden.

Nachhaltigkeit als Resilienz der Nutzungssysteme innerhalb bestimmter Leitplanken aufzufassen, entspricht in wesentlichen Teilen dem von Daly (1973) eingeführten Konzept der ,starken Nachhaltigkeit“. Hierbei wird davon ausgegangen, dass sich das Naturkapital, das heißt Lebensräume, Arten, Stoffkreisläufe etc., in Zukunft nicht beliebig durch technische Entwicklungen substituieren lässt (Ott/ Döring 2008). Im Rahmen einer „starken Nachhaltigkeit“ soll es daher konstant gehalten werden, unter anderem dadurch, dass erneuerbare Ressourcen nur in dem Maße genutzt werden, in dem sie sich regenerieren (von Egan-Krieger/Ott/Voget 2007: 13).

\subsection{Indikatoren- und Managementsysteme nachhaltiger land- und forstwirtschaftlicher Nutzung}

Seit mehreren Jahrzehnten werden ein unzureichendes Monitoring, eine verbesserungsbedürftige Erfolgskontrolle und ein ungenügendes Wirkungsverständnis von Naturschutzmaßnahmen festgestellt (Bürger/Dröschmeister 2001; Marquard/Vohland 2012). Unklare, oft auch widersprüchliche Ziele des Naturschutzes, ein uneffektives
Informationsmanagement, lange Betrachtungszeiträume, zu geringe Ressourcen und eine fehlende Bereitschaft zur Evaluation sind die wichtigsten Ursachen hierfür (Kapos/ Balmford/Aveling et al. 2008). Die eigene Analyse (vgl. Kapitel 2.5) der gegenwärtig auf der nationalen Ebene etablierten Indikatorensysteme deutet ebenfalls auf erhebliche Defizite hin.

Allerdings muss festgehalten werden, dass die Entwicklung der Indikatorensysteme in den letzten Jahrzehnten stark vorangetrieben wurde und so seit den 1990er-Jahren erhebliche Fortschritte auf dem Weg zur Bewertung nachhaltiger Landnutzung erreicht worden sind. Zudem stehen durch repräsentative Inventuren wie die Bundeswaldinventur (BMEL 2016) und die Digitalisierung flächendeckender Kartierungen mittlerweile umfangreiche raumbezogene Daten zu Verfügung. Der Aufbau eines befriedigenden Indikatorensystems zur Bewertung der Nachhaltigkeit land- und forstwirtschaftlicher Nutzung stellt eine sehr anspruchsvolle Aufgabe dar. Dies zeigen auch die Erfahrungen mit anderen nationalen Monitoringprogrammen der biologischen Vielfalt (BAFU 2014).

Zusammenfassend sind die folgenden Anforderungen an ein Indikatorensystem zur Bewertung der Nachhaltigkeit der land- und forstwirtschaftlichen Nutzung im Hinblick auf die biologische Vielfalt zu stellen (vgl. auch Arndt/ Nobel/Schweizer 1987; Blab 1988): Die einzelnen Indikatoren sollten alle relevanten Ebenen der biologischen Vielfalt abdecken (genetische Vielfalt, Arten-, Ökosystem- und Landschaftsvielfalt), gemäß dem PSR-System so aussagekräftig in Bezug auf Belastungen, Zustandsveränderungen und Maßnahmen sein, dass ein ausreichendes Wirkungsverständnis möglich wird, reproduzierbar sein und möglichst wirtschaftlich erhoben werden. Das Indikatorensystem sollte gut begründete Grenzwerte für tolerable Zustände aller Indikatoren beinhalten sowie mit Indikatorensystemen auf anderen Skalen (z. B. betriebliche Zertifizierung) und anderer Sektoren (z. B. Wasserwirtschaft) harmonisiert werden.

Die größten Herausforderungen liegen offenbar darin, die Indikatoren sinnvoll zu einem System zu kombinieren, aussagekräftige Grenzwerte abzuleiten und Schnittstellen zwischen den Indikatorensystemen auf unterschiedlichen Skalen und zwischen den Sektoren zu entwickeln. Wie komplex diese Aufgabe ist, wird bei einem Blick auf die Vielzahl an Indikatorensystemen deutlich, die von der betrieblichen über die nationale und europäische bis zur globalen Ebene existieren und kaum miteinander harmonisiert sind. Auch in den deutschen Großschutzgebieten existieren zahlreiche, nur wenig aufeinander abgestimmte Monitoringprogramme (vgl. Kowatsch/Hampicke/Kruse-Graumann et al. 2011). Eine Bündelung und Harmonisierung der Indikatorensysteme erscheint zurzeit eine vordringliche Aufgabe. Hierbei könnten die Großschutzgebiete gemäß ihrem gesetzlichen Auftrag einer einheitlichen Entwick- 
lung (Bundesnaturschutzgesetz in der Fassung vom 29. Juli 2009) eine Modellfunktion übernehmen.

\subsection{Wie nachhaltig ist die land- und forstwirtschaftliche Nutzung?}

Unter Berücksichtigung der Tatsache, dass die bisher etablierten Indikatorensysteme nur eingeschränkte Aussagen erlauben, kann weder Land- noch Forstwirtschaft eine umfassend nachhaltige Nutzung im Hinblick auf die biologische Vielfalt attestiert werden. Auffällig ist allerdings, dass für die Forstwirtschaft gehäuft positive Entwicklungen bei Maßnahmenindikatoren wie der Totholzmenge, der Zertifizierung oder den Schutzgebietsflächen auftreten und sich nur wenige Indikatoren im negativen Bereich bewegen. Wird Nachhaltigkeit als Prozess der Annäherung an die Leitidee der generationenübergreifenden Gerechtigkeit aufgefasst, so wird hieran eine nachhaltige Ausrichtung deutlich. Im Unterschied dazu ist eine eindeutige Weiterentwicklung der Landwirtschaft in Richtung nachhaltiger Nutzung im Hinblick auf die biologische Vielfalt nicht erkennbar.

\subsection{Beitrag der Großschutzgebiete zu einer nachhaltigen Landnutzung}

Mit den unterschiedlichen Zielsetzungen der deutschen Großschutzgebiete liegen differenzierte Instrumente der Raumordnung vor, die von ihrem Ansatz her geeignet sind, einen wichtigen Beitrag zur Lösung der Konflikte zwischen Landnutzung und Naturschutz zu erbringen. Während Naturparke und Biosphärenreservate diese Integrationsaufgabe innerhalb des Schutzgebietes wahrnehmen sollen, verfolgen Nationalparke einen überwiegend segregativen Ansatz. Dies erscheint sinnvoll, um die an ungenutzte Landschaften gebundene biologische Vielfalt erhalten bzw. wiederherstellen zu können, was im Fall des Nationalparks Bayerischer Wald offenbar bereits gelingt (Bässler 2014).

Die Flächenanteile und räumliche Verteilung der jeweiligen Großschutzgebietskategorien können bereits als Schritt in Richtung einer differenzierten Landnutzung gedeutet werden. Differenzierte Landnutzung verbindet die Integration von Nutz- und Schutzfunktionen auf großer Fläche mit einem gewissen Anteil an Vorrangflächen (Haber 2014). Diese Mischstrategie erscheint zielführend (Winkel 2008), weil segregative und integrative Naturschutzkonzepte jeweils spezifische Stärken und Schwächen aufweisen (Broggi 1994; Scherfose 2000).

Während die segregativ ausgerichteten Nationalparke den mit Abstand kleinsten Flächenanteil einnehmen und auch der Flächenumfang der Biosphärenreservate mit einen Anteil von 3,7\% gering erscheint, umfassen Naturparke mehr als ein Viertel der Landfläche Deutschlands. Insge- samt decken die Großschutzgebiete zwar die wesentlichen Naturräume Deutschlands ab (Scherfose 2016), sind allerdings nicht repräsentativ über die Großlandschaften verteilt. Schwerpunkträume finden sich in den weniger produktiven und strukturschwachen Regionen. Während der Ausschluss von Nationalparken in Großlandschaften mit hoher Produktionsleistung der Landwirtschaft sinnvoll erscheint, ist der geringe Anteil der integrativ ausgerichteten Großschutzgebietskategorien in diesen Naturräumen kritisch zu sehen und kann als Ausdruck ihrer fehlenden Akzeptanz gedeutet werden.

Die Ausweisung von Schutzgebieten in Deutschland erfolgte über viele Jahrzehnte weder auf der Bundesebene noch auf der Ebene der einzelnen Bundesländer systematisch (Bohn 1992; Scheurlen 2000), obwohl es Ansätze für eine systematische Einrichtung von Nationalparken gegeben hat (Heiss 1992; Diepolder 1997; FÖNAD 1997) und das deutsche Schutzgebietssystem mehrfach analysiert wurde (Ssymank 2000; Blab 2002; Steer/Scherfose/ Balzer 2008; Job 2010). Das Defizit einer systematischen Schutzgebietsplanung ist angesichts der konkreten Ziele der Nationalen Strategie zur Biologischen Vielfalt kritisch. So werden Flächenziele für Wälder mit natürlicher Entwicklung (5\% im gesamten Wald, $10 \%$ im öffentlichen Wald) und Wildnis (2\% der Landesfläche) formuliert (BMU 2007: 31, 40, 45), die bei Weitem noch nicht erreicht sind. Der Anteil an Wäldern mit natürlicher Entwicklung betrug im Jahr 2013 1,9\% der Waldfläche (Engel/Wildmann/Spellmann et al. 2016: 55). Auch der Flächenumfang an Wildnis- bzw. Wildnisentwicklungsgebieten liegt noch deutlich unter dem Zielwert der „Nationalen Strategie zur Biologischen Vielfalt“ (Opitz/Reppin/Schoof et al. 2015: 411; Spellmann/ Engel/Meyer 2015: 415). Für die Ausgestaltung des weiteren Prozesses ist eine systematische Schutzgebietsplanung (vgl. Margules/Pressey 2000) als fachliche Grundlage dringend anzuraten (Meyer/Engel 2016). In diesem Sinne fordert die aktuelle „Naturschutzoffensive 2020“ (BMUB 2015b: 23) einen „Nationalen Aktionsplan Schutzgebiete“ und die Einrichtung eines Biotopverbundsystems.

Inwieweit ein Schutzgebietssystem seine Aufgaben erfüllen kann, hängt neben seiner Repräsentativität und dem Flächenumfang auch entscheidend vom Management ab. In Übereinstimmung mit den bisher vorliegenden Evaluationen von Großschutzgebieten und den Ergebnissen zum Status der biologischen Vielfalt in Deutschland ist hier deutlicher Verbesserungsbedarf festzustellen. Dies wird durch das Ziel der „Naturschutzoffensive 2020“ (BMUB 2015b) unterstrichen, bis 2020 ein gut funktionierendes Managementsystem für alle Großschutzgebiete und Natura 2000Gebiete zu etablieren. Auf der Gebietsebene kristallisieren sich die folgenden Handlungsfelder heraus: 
- Verbesserung von Monitoring und Erfolgskontrolle bei allen drei Großschutzgebieten.

- Weiterentwicklung einer multifunktionalen nachhaltigen Landnutzung in den Biosphärenreservaten und Naturparken, wobei in den Biosphärenreservaten bereits klarere Fortschritte in diese Richtung erkennbar sind.

\section{Schlussfolgerungen}

Unter den heutigen Rahmenbedingungen einer hoch entwickelten, urban geprägten Industriegesellschaft mit veränderten Ansprüchen an Naturschutz und Landnutzung, der anstehenden Energiewende, einer flächendeckenden Eutrophierung durch Stickstoffeinträge und dem drohenden Klimawandel, ist die Konkretisierung einer nachhaltigen und multifunktionalen Landnutzung in Deutschland dringender denn je. Naturparke und Biosphärenreservate können in diesem Prozess eine zentrale Rolle übernehmen, wenn es gelingt, ihr Management so zu verbessern, dass sie tatsächlich Modellräume für die Integration von Landnutzung und $\mathrm{Na}-$ turschutz darstellen. Bei Nationalparken ist bereits deutlich klarer erkennbar, dass sie ihre Funktion als großflächige Prozessschutzgebiete in naher Zukunft erfüllen werden.

Danksagung Die Autoren möchten Falko Engel für die Berechnung der Flächenanteile der Großschutzgebiete sowie Hermann Spellmann, Hubert Job und Manuel Woltering für wertvolle Kommentare und Hinweise zum Manuskript herzlich danken.

\section{Literatur}

Arndt, U.; Nobel, W.; Schweizer, B. (1987): Bioindikatoren. Möglichkeiten, Grenzen und neue Erkenntnisse. Stuttgart.

Bässler, C. (2014): Prozessschutz und Biodiversität im Nationalpark Bayerischer Wald. In: Scherfose, V. (Hrsg.): Nationalparkmanagement in Deutschland. Bonn, 177-188. = Naturschutz und Biologische Vielfalt 136.

Bässler, C.; Müller, J. (2015): Selbst naturnahe Waldwirtschaft stört biologische Prozesse. In: AFZ/DerWald 70, 3, 42-43.

BAFU - Bundesamt für Umwelt (Hrsg.) (2014): Biodiversitätsmonitoring Schweiz BDM. Beschreibung der Methoden und Indikatoren. Bern.

Becken, S.; Job, H. (2014): Protected areas in an era of global-local change. In: Journal of Sustainable Tourism 22, 4, 507-527.

Beese, F. O. (1996): Indikatoren für eine multifunktionelle Waldnutzung. In: Forstwissenschaftliches Centralblatt 115, 1, 65-79.

BfN - Bundesamt für Naturschutz (2010): Großschutzgebiete in Deutschland - Ziele und Handlungserfordernisse - Positionspapier des Bundesamtes für Naturschutz. Bonn.

Blab, J. (1988): Bioindikatoren und Naturschutzplanung. Theoretische Anmerkungen zu einem komplexen Thema. In: Natur und Landschaft $63,4,147-149$.

Blab, J. (2002): Stellenwert und Rolle von Naturschutzgebieten in Deutschland. In: Natur und Landschaft 77, 8, 333-339.

BMEL - Bundesministerium für Ernährung und Landwirtschaft (2016): Der Wald in Deutschland. Ausgewählte Ergebnisse der dritten Bundeswaldinventur. Berlin.
BMU - Bundesministerium für Umwelt, Naturschutz und Reaktorsicherheit (2007): Nationale Strategie zur biologischen Vielfalt. Bonn.

BMUB - Bundesministerium für Umwelt, Naturschutz, Bau und Reaktorsicherheit (2015a): Indikatorenbericht 2014 zur Nationalen Strategie zur biologischen Vielfalt. Berlin.

BMUB - Bundesministerium für Umwelt, Naturschutz, Bau und Reaktorsicherheit (2015b): Naturschutz-Offensive 2020 - Für biologische Vielfalt. Berlin.

Bohn, U. (1992): Buchen-Naturwaldreservate und Buchenwald-Naturschutzgebiete in Mitteleuropa. Überblick und naturschutzfachliche Bewertung. In: Naturschutzzentrum Nordrhein-Westfalen (Hrsg.): Buchenwaldökosysteme. Naturerbe Mitteleuropas. Recklinghausen, 56-64. = NZ NRW Seminarberichte 12/1992.

Bolte, A. (2015): Alt- und Totholzkonzepte zeigen Wirkung. In: AFZ/Der Wald 70, 6, 37-38.

Bork, J.; Knoke, T. (2015): Weder seltene Arten noch Biodiversität nehmen ab. In: AFZ/DerWald 70, 9, 28-29.

Broggi, M. F. (1994): Strategien der Umsetzung von Naturschutzzielen in der Kulturlandschaft. In: Landesanstalt für Umweltschutz Baden-Württemberg (Hrsg.): 2. Statuskolloquium des PAÖ. Karlsruhe, 107-123. = Veröffentlichungen Projekt „Angewandte Ökologie" 8 .

Bürger, K.; Dröschmeister, R. (2001): Naturschutzorientierte Umweltbeobachtung in Deutschland: Ein Überblick. In: Natur und Landschaft 76, 2, 49-57.

Cardinale, B. J.; Duffy, J. E.; Gonzalez, A.; Hooper, D. U.; Perrings, C.; Venail, P.; Narwani, A.; Mace, G. M.; Tilman, D.; Wardle, D. A.; Kinzig, A. P.; Daily, G. C.; Loreau, M.; Grace, J. B.; Larigauderie, A.; Srivastava, D. S.; Naeem, S. (2012): Biodiversity loss and its impact on humanity. In: Nature 486, 59-67.

Christen, O.; Hövelmann, L.; Hülsbergen, K. J.; Packeiser, M.; Rimpau, J.; Wagner, B. (Hrsg.) (2009): Nachhaltige landwirtschaftliche Produktion in der Wertschöpfungskette Lebensmittel. Berlin. $=$ Initiativen zum Umweltschutz 78 .

Daly, H. E. (1973): Toward a steady-state economy. San Francisco.

Dantsis, T.; Douma, C.; Giourga, C.; Loumou, A.; Polychronaki, E. A. (2010): A methodological approach to assess and compare the sustainability level of agricultural plant production systems. In: Ecological Indicators 10, 2, 256-263.

Deutscher Bundestag (1998): Konzept Nachhaltigkeit. Vom Leitbild zur Umsetzung. Abschlussbericht der Enquete-Kommission „Schutz des Menschen und der Umwelt - Ziele und Rahmenbedingungen einer nachhaltig zukunftsverträglichen Entwicklung“ des 13. Bundestages (Drucksache 13/11200). Bonn.

Diepolder, U. (1997): Zustand der deutschen Nationalparke im Hinblick auf die Anforderungen der IUCN. Dissertation an der Technischen Universität München.

DLG - Deutsche Landwirtschaftliche Gesellschaft (2015): DLGNachhaltigkeitsbericht 2015. Frankfurt am Main.

DRL - Deutscher Rat für Landespflege (Hrsg.) (2010): Biosphärenreservate sind mehr als Schutzgebiete. Wege in eine nachhaltige Zukunft. Meckenheim. $=$ Schriftenreihe des Deutschen Rates für Landespflege 83.

EEA - European Environment Agency (2015): EEA Indicators. http:// www.eea.europa.eu/data-and-maps/indicators\#c $5=\& \mathrm{c} 0=10 \& \mathrm{~b}_{-}$ start $=0$ (14.07.2016).

Ellis, E. C.; Ramankutty, N. (2008): Putting people in the map: anthropogenic biomes of the world. In: Frontiers in Ecology and the Environment 6, 8, 439-447.

Engel, F.; Wildmann, S.; Spellmann, H.; Reif, A.; Schultze, J. (2016): Bilanzierung der nutzungsfreien Wälder in Deutschland. In: Engel, F.; Bauhus, J.; Gärtner, S.; Kühn, A.; Meyer, P.; Reif, A.; Schmidt, M.; Schultze, J.; Späth, V.; Stübner, S.; Wildmann, S.; Spellmann, H. (Hrsg.): Wälder mit natürlicher Entwicklung in Deutschland: Bilanzierung und Bewertung. Bonn, 37-74. = Naturschutz und Biologische Vielfalt 145. 
FÖNAD - Föderation der Natur- und Nationalparke Europas (1997): Studie über bestehende und potentielle Nationalparke in Deutschland. Bonn. = Angewandte Landschaftsökologie 10.

Forest Europe Liaison Unit Oslo (2011): State of Europe's Forests 2011. Status and Trends in Sustainable Forest Management in Europe. Oslo.

Forst, R.; Scherfose, V. (2010): Entwicklungen und Perspektiven deutscher Naturparks. In: Forst, R.; Scherfose, V. (Hrsg.): Naturschutzmaßnahmen und -aktivitäten in den deutschen Naturparken. Bonn, 189-195. = Naturschutz und Biologische Vielfalt 104.

Gehrlein, U.; Kullmann, A. (2011): Strategien für nachhaltiges Wirtschaften in Biosphärenreservaten. In: Natur und Landschaft 86, 7, 312-317.

Haber, W. (2004): Über den Umgang mit Biodiversität. In: Berichte der Bayerischen Akademie für Naturschutz und Landschaftspflege $28,25-43$.

Haber W. (2014): Landwirtschaft und Naturschutz. Weinheim.

Hager, J. (2009): Naturparke und ihre Rolle für den Erhalt von Kulturlandschaften und deren Biodiversität. In: EUROPARC Deutschland (Hrsg.): Nationale Naturlandschaften und Biodiversität. Vielfalt macht stark! Berlin, 14-17.

Hayati, D.; Ranjbar, Z.; Karami, E. (2010): Measuring Agricultural Sustainability. In: Lichtfouse, E. (Hrsg.): Biodiversity, biofuels, agroforestry and conservation agriculture. Berlin, 73-100. = Sustainable Agriculture Reviews 5.

Heiland, S.; Hoffmann, A. (2013): Erste Evaluierung der deutschen Nationalparks: Erfahrungen und Ergebnisse. In: Natur und Landschaft 88, 7, 303-308.

Heiss, G. (1992): Erfassung und Bewertung großflächiger Waldgebiete zum Aufbau eines Schutzgebietssystems in der Bundesrepublik Deutschland. München. $=$ Forstliche Forschungsberichte München 120.

Holling, C. S. (1973): Resilience and Stability of Ecological Systems. In: Annual Review of Ecology and Systematics 4, 1-23.

Hoogstra-Klein, M. A.; Burger, M. (2013): Rational versus adaptive forest management planning: exploratory research on the strategic planning practices of Dutch forest management organizations. In: European Journal of Forest Research 132, 5, 707-716.

Hooper, D. U.; Chapin, F. S.; Ewel, J. J.; Hector, A.; Inchausti, P.; Lavorel, S.; Lawton, J. H.; Lodge, D. M.; Loreau, M.; Naeem, S.; Schmid, B.; Setälä, H.; Symstad, A. J.; Vandermeer, J.; Wardle, D. A. (2005): Effects of biodiversity on ecosystem functioning: A consensus of Current Knowledge. In: Ecological Monographs 75, $1,3-35$.

Hurni, H. (2000): Assessing sustainable land management (SLM). In: Agriculture, Ecosystems and Environment 81, 2, 83-92.

Jedicke, E. (2013): Bericht zur Überprüfung des UNESCO-Biosphärenreservats Rhön 2013. München, Wiesbaden, Erfurt.

Job, H. (2010): Welche Nationalparke braucht Deutschland? In: Raumforschung und Raumordnung 68, 2, 75-89.

Job, H. (2016): Großschutzgebiete. In: Akademie für Raumforschung und Landesplanung - ARL (Hrsg.): Handwörterbuch zur Stadtund Raumentwicklung. Hannover (im Druck).

Job, H.; Harrer, B.; Metzler, D.; Hajizadeh-Alamdary, D. (2005): Ökonomische Effekte von Großschutzgebieten. Untersuchung der Bedeutung von Großschutzgebieten für den Tourismus und die wirtschaftliche Entwicklung der Region. Bonn. = BfN-Skripten 135.

Job, H.; Kraus, F.; Merlin, C.; Woltering, M. (2013): Wirtschaftliche Effekte des Tourismus in Biosphärenreservaten Deutschlands. Bonn. $=$ Naturschutz und Biologische Vielfalt 134.

Job, H.; Woltering, M.; Harrer, B. (2009): Regionalökonomische Effekte des Tourismus in deutschen Nationalparken. Bonn. = Naturschutz und biologische Vielfalt 76.

Kapos, V.; Balmford, A.; Aveling, R.; Bubb, P.; Carey, P.; Entwistle, A.; Hopkins, J.; Mulliken, T.; Safford, R.; Stattersfield, A.; Walpole, M.; Manica, A. (2008): Calibrating conservation: new tools for measuring success. In: Conservation Letters 1, 4, 155-164.
Kowatsch, A.; Hampicke, U.; Kruse-Graumann, L.; Plachter, H. (2011): Indikatoren für ein integratives Monitoring in deutschen Großschutzgebieten. Bonn. = BfN-Skripten 302.

Kraus, F.; Merlin, C.; Job, H. (2014): Biosphere Reserves and their Contribution to Sustainable Development: A Value-Chain Analysis in the Rhön Biosphere Reserve, Germany. In: Zeitschrift für Wirtschaftsgeographie 58, 1, 164-180.

Lamprecht, H. (1970): Uber allgemein gültige Grundlagen im Waldbau von heute und morgen. In: Forstarchiv 41, 10, 199-205.

Lindenmayer, D.; Cunningham, S.; Young, A. (Hrsg.) (2012): Land Use Intensification. Effects on Agriculture, Biodiversity and Ecological Processes. Collingwood.

Lindenmayer, D.; Hunter, M. (2010): Some Guiding Concepts for Conservation Biology. In: Conservation Biology 24, 6, 1459-1468.

Margules, C. R.; Pressey, R. L. (2000): Systematic conservation planning. In: Nature 405, 243-253.

Marquard, E.; Vohland, K. (2012): Nationales Biodiversitätsmonitoring 2020. Zusammenfassender Bericht des Workshops vom 14. und 15. Juni 2012 in Berlin. Berlin.

MEA - Millennium Ecosystem Assessment Board (2005): Ecosystems and human well-being: synthesis. Washington D. C.

Meyer, P. (2013): Biodiversität im Wald. In: AFZ/Der Wald 68, 17, $24-25$.

Meyer, P., Engel, F. (2016): Repräsentanz der Wälder mit natürlicher Entwicklung in Deutschland. In: Engel, F.; Bauhus, J.; Gärtner, S.; Kühn, A.; Meyer, P.; Reif, A.; Schmidt, M.; Schultze, J.; Späth, V.; Stübner, S.; Wildmann, S.; Spellmann, H. (Hrsg.): Wälder mit natürlicher Entwicklung in Deutschland: Bilanzierung und $\mathrm{Be}-$ wertung. Bonn, 151-190. = Naturschutz und Biologische Vielfalt 145.

Meyer, S.; Wesche, K.; Krause, B.; Brütting, C.; Hensen, I.; Leuschner, C. (2014): Diversitätsverluste und floristischer Wandel im Ackerland seit 1950. In: Natur und Landschaft 89, 9/10, 392-398.

Opitz, S.; Reppin, N.; Schoof, N.; Drobnik, J.; Finck, P.; Riecken, U.; Mengel, A.; Reif, A.; Rosenthal, G. (2015): Wildnis in Deutschland. Nationale Ziele, Status Quo und Potenziale. In: Natur und Landschaft 90, 9/10, 406-412.

Ostrom, E. (2009): A General Framework for Analyzing Sustainability of Social-Ecological System. In: Science 325, 419-422.

Ott, K.; Döring, R. (2008): Theorie und Praxis starker Nachhaltigkeit. Marburg. $=$ Beiträge zur Theorie und Praxis starker Nachhaltigkeit 1.

Papworth, S. K.; Rist, J.; Coad, L.; Milner-Gulland, E. J. (2009): Evidence for shifting baseline syndrome in conservation. In: Conservation Letters 2, 2, 93-100.

Pauly, D. (1995): Anecdotes and the shifting baseline syndrome of fisheries. In: Trends in Ecology and Evolution 10, 10, 430.

Pereira, H. M.; Navarro, L. M.; Martins, I. S. (2012): Global biodiversity change: The bad, the good, and the unknown. In: Annual Review of Environment and Resources 37, 25-50.

Pirkl, A.; Riedel, B. (1991): Indikatoren und Zielartensysteme in der Naturschutz- und Landschaftsplanung. In: Henle, K.; Kaule, G. (Hrsg.): Arten- und Biotopschutzforschung für Deutschland. Jülich, 343-346. = Berichte aus der ökologischen Forschung 4.

Puettmann, K. J.; Messier, C.; Coates, K. D. (2014): Managing forests as complex adaptive systems: introductory concepts and applications. In: Messier, C.; Puettmann, K. J.; Coates, K. D. (Hrsg.): Managing forests as complex adaptive systems. London, New York, $3-16$.

Reidsma, P.; Tekelenburg, T.; van den Berg, M.; Alkemade, R. (2006): Impacts of land-use change on biodiversity: An assessment of agricultural biodiversity in the European Union. In: Agriculture, Ecosystems and Environment 114, 1, 86-102.

Schanz, H. (1995): Forstliche Nachhaltigkeit. Befragung zum Begriffsverständnis der Forstleute in Deutschland. In: AFZ/DerWald 52, 4, 188-192.

Scherfose, V. (2000): Naturschutzfachplanungen und ihre raumordnerische Umsetzung am Beispiel der Naturschutzgroßprojekte des 
Bundes. In: Ssymank, A. (Hrsg.): Vorrangflächen, Schutzgebietssysteme und naturschutzfachliche Bewertung großer Räume in Deutschland. Bonn, 215-239. = Schriftenreihe für Landschaftspflege und Naturschutz 63.

Scherfose, V. (2016): What role can parks play for sustainable development in Germany? In: Hammer, T.; Mose, I.; Siegrist, D.; Weixlbaumer, N. (Hrsg.): Parks of the Future. München, 191-209.

Scheurlen, K. (2000): Situationsanalyse bestehender Schutzgebietssysteme am Beispiel von Naturschutzgebieten. In: Ssymank, A. (Hrsg.): Vorrangflächen, Schutzgebietssysteme und naturschutzfachliche Bewertung großer Räume in Deutschland. Bonn, 127-146. $=$ Schriftenreihe für Landschaftspflege und Naturschutz 63.

Schneider, T. W. (1995): Kriterien und Indikatoren für eine nachhaltige Bewirtschaftung der Wälder. In: AFZ/DerWald 50, 4, 184-187.

Schrader, N. (2006): Die deutschen Biosphärenreservate auf dem Prüfstand! Dissertation an der Universität Trier.

Schrader, N. (2010): Biosphärenreservate auf dem Prüfstand - Ergebnisse einer ersten unabhängigen Evaluierung. In: Deutscher Rat für Landespflege - DRL (Hrsg.): Biosphärenreservate sind mehr als Schutzgebiete. Wege in eine nachhaltige Zukunft. Meckenheim, 86-89. = Schriftenreihe des Deutschen Rates für Landespflege 83 .

Schumacher, H.; Job, H. (2013): Nationalparks in Deutschland. Analyse und Prognose. In: Natur und Landschaft 88, 7, 309-314.

Spellmann, H. (2003): Sicherung einer nachhaltigen Waldentwicklung auf überbetrieblichen Ebenen. In: Forstwissenschaftliches Centralblatt $122,4,250-257$.

Spellmann, H. (2013): 300 Jahre forstliche Nachhaltigkeit. In: AFZ/Der Wald 68, 11, 14-18.

Spellmann, H.; Engel, F.; Meyer, P. (2015): Natürliche Waldentwicklung auf 5\% der Waldfläche. In: Natur und Landschaft 90, 9/10, $413-416$.

Ssymank, A. (2000): Rahmenbedingungen für die naturschutzfachliche Bewertung großer Räume und fachliche Anforderungen an ein Bundesvorrangflächensystem für den Naturschutz. In: Ssymank, A. (Hrsg.): Vorrangflächen, Schutzgebietssysteme und naturschutzfachliche Bewertung großer Räume in Deutschland. Bonn, 11-47. = Schriftenreihe für Landschaftspflege und Naturschutz 63 .

Steer, U.; Scherfose, V.; Balzer, S. (2008): Ausgewählte Aspekte des deutschen Schutzgebietssystems. In: Natur und Landschaft 83, 3, 93-100.

Stoate, C.; Boatman, N. D.; Borralho, R. J.; Carvalho, C. R.; de Snoo, G. R.; Eden, P. (2001): Ecological impacts of arable intensification in Europe. In: Journal of Environmental Management 63, 4, 337-365.

Ulrich, B. (1991): Folgerungen aus 10 Jahren Waldökosystem- und Waldschadensforschung. In: Forst und Holz 46, 21, 575-588.

UNESCO (2013): Bericht zur Überprüfung des UNESCO-Biosphärenreservates Südost-Rügen. Putbus.

von Egan-Krieger, T.; Ott, K.; Voget, L. (2007): Der Schutz des Naturerbes. In: Aus Politik und Zeitgeschichte 24, 10-17.

Wagner, S. (2004): Möglichkeiten und Beschränkungen eines funktionsorientierten Waldbaus. In: Forst und Holz 59, 3, 105-111.

Walter, C.; Stützel, H. (2009): A new method for assessing the sustainability of land-use systems (I): Identifying the Relevant Issues. In: Ecological Economics 68, 5, 1275-1287.

WCED - World Commission on Environment and Development (1987): Report of the World Commission on Environment and Development: Our Common Future. Oxford.

Weith, T.; Besendörfer, C.; Gaasch, N.; Kaiser, D. B.; Müller, K.; Repp, A.; Rogga, S.; Strauß, C; Zscheischler, J. (2013): Nachhaltiges Landmanagement: Was ist das? Müncheberg. = Nachhaltiges Landmanagement, Diskussionspapier 7. http:// modul-b.nachhaltiges-landmanagement.de/de/begleitvorhaben/ diskussionspapiere/ (14.07.2016).

Winkel, G. (2008): Schutz von Wäldern außerhalb von Schutzgebieten. Zur Rolle von Segregation und Integration im globalen Waldnaturschutz. In: Natur und Landschaft 83, 4, 162-163.

Wesche, K.; Krause, B.; Culmsee, H.; Leuschner, C. (2012): Fifty years of change in Central European grassland vegetation: Large losses in species richness and animal-pollinated plants. In: Biological Conservation 150, 1, 76-85. 